\title{
CRISPR/CAS-mediated deletion of the upstream regulatory sequences enhances the estrogen-independent expression of chicken ovalbumin
}

\author{
Sara Yousefi Taemeh ${ }^{1}$, Nima Dehdilani ${ }^{1}$, Lena Goshayeshi ${ }^{1}$, Jalil Merhzad ${ }^{2}$, and Hesam \\ Dehghani $^{1}$ \\ ${ }^{1}$ Ferdowsi University of Mashhad Faculty of Veterinary Medicine \\ ${ }^{2}$ University of Tehran Faculty of Veterinary Medicine
}

May 11, 2020

\begin{abstract}
Avian transgenesis has served as a suitable approach to generate bioreactors for the manufacturing of recombinant proteins. Production in chicken cells comes with significant advantages over other systems including providing the human-like glycosylation on target proteins. In this regard, the oviduct-specific ovalbumin promoter has been one of the ideal candidates to drive the expression of transgenes. Previous plasmid-based studies on the regulatory sequences of ovalbumin promoter have led researchers to exploit ovalbumin regulatory elements out of their native genomic context (ex situ) to direct transgene expression in the transgenic chicken bioreactors. Although the inherent limitations on the ex situ use of ovalbumin promoter have promoted the use of native ovalbumin promoter for the expression of a transgene, generation of transgenic chicken is relatively difficult, inefficient, and time-consuming. To overcome these obstacles, in this study we show that CRISPR-mediated deletion of some distal ovalbumin promoter sequences in a non-oviduct cell can lead to the significant expression of the ovalbumin gene, and also a knocked-in reporter, in an estrogen-independent manner. These findings overcome the limitation of cloned promoters, where the promoter regulatory sequences have to be taken out of their cis context and also their native spatial nuclear organization into a plasmid.
\end{abstract}

\section{INTRODUCTION}

In recent decades, the global demand for therapeutic proteins has considerably increased. Although there are various platforms for the production of recombinant proteins, the avian production system, due to its significant advantages over other systems, has been taken into more consideration (Demain \& Vaishnav, 2009; Houdebine, 2018; Ivarie, 2003, 2006; Maksimenko, Deykin, Khodarovich, \& Georgiev, 2013; Raju, Briggs, Borge, \& Jones, 2000). Recombinant human proteins produced in chicken cells in comparison to the produced proteins in plant, bacterial, or non-human mammalian cells, contain posttranslational modifications (including glycosylation) that are much more similar to those in proteins produced by human cells (Zhu et al., 2005). The proper posttranslational modifications are essential for successful therapeutic efficacy, prevention of unintended immune response, the long half-life of proteins, and biological activity in vivo (Elliott et al., 2004; Kodama et al., 2008; Rapp, Harvey, Speksnijder, Hu, \& Ivarie, 2003).

Oligosaccharides on IgG in human and chicken are similar and comprise mainly $\mathrm{N}$-acetylneuraminic acid (NANA), whereas other species contain N-glycolylneuraminic acid (NGNA) and NANA (e.g. rabbit), or only NGNA (e.g. sheep, goats, and cow) (Simon G. Lillico, McGrew, Sherman, \& Sang, 2005; Raju et al., 
2000; T. Shantha Raju, 2003). Therefore, avian expression systems represent desirable platforms for the production of recombinant human proteins.

In the early attempts to produce foreign proteins in avian systems, viral vectors containing a constitutive promoter (such as CVM promoter) were used to drive the expression. The use of these constitutive strong promoters had several disadvantages including variations in the level of protein expression, improper folding of the protein product, the possibility of promoter silencing, and toxicity due to their expression in a wide range of tissues. Thus, there has been an increasing trend toward the use of regulated promoters. Among these, the native hormone-dependent promoters have demonstrated to be efficient in transgene expression. For example, the ovalbumin promoter with its regulatory sequences has been used in the cultured primary oviduct cells or in the transgenic chickens for the production of exogenous proteins (Byun et al., 2011; Cao et al., 2015; Herron et al., 2018; Kodama et al., 2012; M. S. Kwon et al., 2018; S. C. Kwon et al., 2010; S G Lillico et al., 2007; T. Liu et al., 2015; Oishi, Yoshii, Miyahara, \& Tagami, 2018; T. S. Park et al., 2015; Zhu et al., 2005). Despite the successful achievements and progress in this field, primary oviduct cell culture is difficult to perform and needs experimental requirements including efficient transfection of the primary cells, cell-cell and cell-substratum interactions, and dependency to steroid hormones (Yoshimura \& Oka, 1990). Furthermore, the generation of transgenic chicken is elaborate, time-consuming, and requires highly skilled personnel for embryo-manipulation.

Hence, using a hormone-independent promoter to drive the transgene expression in a chicken cell line might be an easy and quick alternative method of production platform for human proteins. This system would allow us to use the significant advantages of production in chicken cells.

The regulatory sequences of the ovalbumin promoter, which has been extensively used in the chicken production systems are well-characterized (Dougherty, Park, \& Sanders, 2009; Dougherty \& Sanders, 2005; Haecker, Muramatsu, Sensenbaugh, \& Sanders, 1995; Kato et al., 1992; Kaye, Bellard, Dretzen, Bellard, \& Chambon, 1984; Kaye et al., 1986; Monroe \& Sanders, 2000; H. M. Park, Haecker, Hagen, \& Sanders, 2000; Sanders \& McKnight, 1988; Schimke, McKnight, Shapiro, Sullivan, \& Palacios, 1975; Schweers, Frank, Weigel, \& Sanders, 1990; Sensenbaugh \& Sanders, 1999; Wang et al., 1989). Although, there are many reports on the cis-acting regulatory sequences responsible for ovalbumin gene expression, other factors including transacting regulatory elements, nucleosomal rearrangements, histone modifications, the chromosomal structure of the gene locus, and the three-dimensional (3D) nuclear organization may play critical roles for its proper expression in oviduct cells (Bellard, Dretzen, Bellard, Oudet, \& Chambon, 1982). Previous plasmid-based studies have shown that the deletion of the steroid-dependent regulatory element (SDRE; -900 to -732) and negative regulatory element (NRE; -308 to -88), as well as the linker between them in the ovalbumin promoter, increases the reporter gene activity driven by that (Haecker et al., 1995; Sanders \& McKnight, 1988; Sensenbaugh \& Sanders, 1999). These reports demonstrated that the presence of ovalbumin proximal promoter $(-87$ to +9$)$ is sufficient for an estroid-independent expression. This led us to hypothesize that thein situ deletions of SDRE and NRE elements in the genome of a chicken non-oviduct cell line may also lead to the steroid-independent ovalbumin gene upregulation, while the trans-acting regulatory elements are still able to exert their effects.

In this study, we have deleted the ovalbumin distal promoter including SDRE and NRE elements in the genome of the DF1 fibroblast cell line via CRISPR/Cas9 system, and have analyzed the increased expression of theOvalbumin gene and the induced activity of an inserted transgene.

\section{MATERIALS AND METHODS}

\subsection{Plasmid construction}

The plasmids expressing Cas9 and sgRNAs targeted to ovalbumin distal promoter (px459-14 \& px45915) and that targeted to ovalbumin exon 2 (px459-6) were designed using the CRISPR design tool 
(http://crispr.mit.edu/) and then generated by routine subcloning techniques (Table 1). The donor vector for CRISPR HDR (homology-directed repair) (pHD_4520) was generated by ligating a PCR-amplified, 556 bp DNA ovalbumin fragment (beginning of the exon 2) as the 5' homology arm, and a PCR-amplified, 526 bp DNA ovalbumin fragment as the 3 ' homology arm (Table 1).

\subsection{Targeted deletion of ovalbumin promoter in cultured DF1 cells}

DF1 cells were received as a generous gift from Dr. Bertrand Pain (The Stem cell and Brain Research Institute, Lyon, France), and cultured as recommended by the ATCC. In order to perform CRISPR excision of ovalbumin promoter with dual sgRNAs, DF1 cells were transfected with px459-14 and px459-15 (Table 1) using Lipofectamine 3000 (Invitrogen, USA), as described previously (Abu-Bonsrah, Zhang, \& Newgreen, 2016). Briefly, $0.5 \mu \mathrm{g}$ from each of px459-14 and px459-15 plasmids were diluted with $50 \mu \mathrm{l}$ OPTI-MEM + GlutaMax (Thermo Fisher Scientific, USA) and was mixed with $50 \mu$ l OPTI-MEM + GlutaMax containing $1 \mu$ Lipofectamine 3000 reagent and then incubated with $0.1-0.15 \times 10^{6}$ DF1 cells for 4 hours. Subsequently, the cells were cultured in $500 \mu \mathrm{l}$ of an antibiotic-free DMEM-F12 culture medium (Thermo Fisher Scientific) and incubated for $24 \mathrm{~h}$ at $38^{\circ} \mathrm{C}$ in a $7.5 \% \mathrm{CO}_{2}$ environment. The transfectd $\mathrm{DF} 1$ cells were exposed to puromycin dihydrochloride $(2.5 \mu \mathrm{g} / \mathrm{ml}$; Sigma-Aldrich, USA) for 3 days. DF1 cells after antibiotic exposure were expanded for 2 to 3 weeks. A mixed population of these cells was subjected to genomic PCR to confirm the deletion of ovalbumin distal promoter in a fraction of cells. After single-cell isolation and clonal expansion, 3 clones of distal ovalbumin promoter knockout DF1 cells (DF1 +/OVA Pro [?] $)$ were acquired that showed correct deletion in the ovalbumin distal promoter by PCR. These 3 clones were analyzed for the expression of ovalbumin by RT-qPCR (see below).

\subsection{Confirmation of the ovalbumin distal promoter deletion}

Genomic DNA was extracted from wild-type and distal ovalbumin promoter knockout DF1 cells (DF1 +/OVA Pro [?] ) using the Genomic DNA Extraction Kit (DENAzist Asia Co., Iran). Gene-targeting events were detected by a single-round or nested PCR using the designed primers (Table 2) and Taq DNA polymerase master mix RED (Ampliqon, Denmark), and Sanger sequencing of the amplicons (Genomin Co., Iran).

\subsection{Analysis of Ovalbumin expression in DF1 cells with the deletion of distal ovalbumin pro- moter}

Total RNA was isolated from magnum tissue (from a 35-week old laying hen), wild-type, and distal ovalbumin promoter knockout DF1 cells (DF1+/OVA Pro [?]) using the Total RNA Isolation Kit (DENAzist Asia, Iran). The quality and quantity of the extracted RNA were evaluated using gel electrophoresis and a 2000 Nanodrop spectrophotometer (Thermo Scientific, USA). One $\mu \mathrm{g}$ of total RNA was reverse transcribed using random hexamer primers and MMLV reverse transcriptase (Thermo Fisher Scientific, USA). To quantify the level of transcripts for Ovalbumin and Gapdh genes, quantitative RT-PCR reactions containing 1x SYBR Green Real-time PCR Master Mix (Thermo Scientific, USA), $2 \mu \mathrm{l}$ cDNA template and each primer (Table 2, Supplementary Figure 3) at $250 \mathrm{nM}$ in a $20 \mu \mathrm{l}$ reaction volume, were carried out in a Rotor-Gene Q real-time PCR cycler (Qiagen, USA). Amplification steps were: $95^{\circ} \mathrm{C}$ for $15 \mathrm{~min}$, followed by 35 cycles of $95^{\circ} \mathrm{C}$ for 30 $\mathrm{s}, 58^{\circ} \mathrm{C}$ for $30 \mathrm{~s}$, and $72^{\circ} \mathrm{C}$ for $30 \mathrm{~s}$. To derive the melting curve, the temperature was increased in steps of $0.2^{\circ} \mathrm{C}$ for $5 \mathrm{~s}$ from $55^{\circ} \mathrm{C}$ to $95^{\circ} \mathrm{C}$. To confirm the identity of products, PCR-amplified bands after clean-up and reaction recovery (DENAzist Asia Co., Iran), were subjected to Sanger sequencing (Genomin Co., Iran).

To adjust the reaction temperature, to find the best concentration of primers, and to optimize the amplification and melting curves (Supplementary Figure 1A), qPCR reactions were repeated. The identity of qPCR products (Supplementary Figure 1B) was confirmed by Sanger sequencing (Genomin Co., Iran). Complementary DNA from the magnum of the 35-week-old hen was serially diluted and subjected to qPCR to make standard curves (Supplementary Figure 2). Each dilution was subjected to the real-time readings in triplicate. Then, the $\log _{10}$ of cDNA concentration for the Ovalbumin and Gapdh genes were plotted against 
the cycle threshold (Ct) numbers to make a standard curve (Supplementary Figure 2). To calculate the reaction efficiency, the slope of standard curves was used in the equation of $\mathrm{E}=(10-1 /$ slope- 1$) \times 100 \%$. Both standard curves were linear in the analyzed range with an acceptable correlation coefficient (R2) (Supplementary Figure 2). Gene expression ratio for the Ovalbumingene over Gapdh was calculated for the magnum, wild-type DF1, and DF1 cell with deletion of distal ovalbumin promoter using the Pfaffl method of relative quantification (Pfaffl, 2001).

\subsection{Targeted reporter knockin in DF1 cells with the deletion of distal ovalbumin promoter}

DF1 +/OvA Pro [?] cells were transfected with px4596 and pHD_4520 (donor reporter vector) using Lipofectamine 3000 (Invitrogen, USA), as described above. The cells $48 \mathrm{hr}$ after transfection were subjected to antibiotic selection with puromycin dihydrochloride $(2.5 \mu \mathrm{g} / \mathrm{ml}$; Sigma-Aldrich, USA). To confirm knockin of the reporter construct (DsRed2-CMV-Puro-IRES-EGFP), genomic PCR and Sanger sequencing (Genomin Co., Iran) were performed. Cells with the inserted reporter and deleted ovalbumin promoter (DF1 +/OVA Pro [?]-Tg (promoterless dsRed) ) were observed and photographed by fluorescence microscopy (Nikon Eclipse Ts2R, Japan) 2 weeks after transfection.

\section{RESULTS}

\subsection{Deletion of the ovalbumin distal promoter elements in DF1 cells via CRISPR/Cas9 system}

Previous studies have shown that the cloning of proximal segment of the ovalbumin promoter lacking the major regulatory sequences of SDRE and NRE is able to increase the chloramphenicol acetyltransferase (CAT) activity on a plasmid construct in LMH cells and primary oviduct cells (Haecker et al., 1995; Monroe \& Sanders, 2000). This activity was not dependent on the presence of estrogen. However, the observed increased activity was up to 17 -fold. Thus, we decided to delete SDRE and NRE elements from the native promoter in the genome of a non-oviduct cell. To this end, we used CRISPR excision strategy to delete regulatory elements from the ovalbumin promoter in DF1 fibroblast cells (Figure 1A). Cells with the deleted promoter segments were subjected to genomic PCR and Sanger sequencing to confirm the deletion (Figures $1 \mathrm{~B}$ and 1C). Then, 288 cells were individually grown to acquire clones with homogenous population of cells.

\subsection{Deletion of the ovalbumin distal promoter elements induces the expression of ovalbumin mRNA}

We asked whether the deletion of SDRE and NRE elements from the native promoter in the genome of a non-oviduct cell would be able to increase the Ovalbumin transcription. Thus, three individually grown DF1 clones (from 288 clones) with the deletion of SDRE and NRE elements were analyzed for the expression of Ovalbumin by RT-qPCR. In DF1 cells lacking the SDRE, linker and NRE segments in their ovalbumin promoter and grown in vitro in the absence of estrogen, the transcript levels of Ovalbumin increased to more than $10^{4}$-fold compared to that in the wild-type DF1 cell. The transcript levels of Ovalbumin in the hormonally-activated tissue of magnum from 35 -week old laying hen was $10^{7}$-fold compared to that in the wild-type DF1 cells (Figure 2).

3.3. The inserted fluorescent reporter is activated under the control of the ovalbumin promoter with the deletion of distal elements

Next, we tried to assess the functionality of the distally-deleted ovalbumin promoter in DF1 cells to activate a foreign transgene. For this purpose, we designed a construct including a promoterless reporter and used CRISPR HDR to insert it in the exon 2 of the Ovalbumin gene (125 bp after ATG) (Figure 3A). The insertion of this reporter was confirmed by genomic PCR, Sanger sequencing, and fluorescence microscopy 
for GFP (Figures 3B, 3C, and the left panel in 3D). The promoterless DsRed2 reporter under the function of distally-deleted ovalbumin promoter became activated and its red fluorescence was imaged by fluorescence microscopy (Figure 3D). This experiment confirmed that a non-oviduct chicken cell with the deletion of distal elements of ovalbumin promoter can express an inserted transgene in an estrogen-independent manner.

\section{DISCUSSION}

We have shown that CRISPR-mediated deletion of distal ovalbumin promoter in DF1 cells (DF1 +/OvA Pro [?]) induces the expression of Ovalbumin mRNA (Figures 1,2). In addition, in cells with this kind of promoter, we inserted a promoterless reporter in theOvalbumin gene (DF1 +/OVA Pro [?]-Tg (promoterless dsRed) ) and registered the expression of the reporter protein (DsRed2 fluorescence) (Figure 3). In this study, we showed that a chicken non-oviduct cell line with deletion of distal promoter sequences can serve as a chicken production model for steroid-independent expression of a transgene driven by endogenous ovalbumin promoter (Figure $4)$.

Transgenesis has become an important technique for generating biopharmaceutical products. The application of effective promoters is essential for achieving high expression levels and well-structured recombinant proteins. Although constitutive strong promoters have been extensively used to drive the expression of transgenes, they increase the metabolic burden of host cells, resulting in cell debilitation and cell population reduction in culture. Utilization of constitutive strong promoters might also lead to toxicity for the host cell due to the activation of unfolded protein response and aggregation of misfolded proteins in the host cells (Z. Liu, Tyo, Martinez, Petranovic, \& Nielsen, 2012). In this regard, researchers have tried to discover the proper promoters for the continuous production of recombinant proteins at a convenient rate, exclusive to a preferred cell kind, and with appropriate posttranslational modifications and proper protein folding.

Tissue-specific ovalbumin promoter has been one of the novel candidates for the large-scale production of pharmaceutical proteins. The synthesis of several therapeutic proteins under the control of regulatory sequences from the chicken Ovalbumin gene has been reported (Byun et al., 2011; Cao et al., 2015; Herron et al., 2018; Kodama et al., 2012; M. S. Kwon et al., 2018; S. C. Kwon et al., 2010; S G Lillico et al., 2007; T. Liu et al., 2015; Oishi et al., 2018; T. S. Park et al., 2015; Zhu et al., 2005). Although the regulatory elements in the Ovalbumin gene are well characterized out of their genomic context (Dougherty et al., 2009; Dougherty \& Sanders, 2005; Haecker et al., 1995; Kato et al., 1992; Kaye et al., 1984, 1986; Monroe \& Sanders, 2000; H. M. Park et al., 2000; Sanders \& McKnight, 1988; Schimke et al., 1975; Schweers et al., 1990; Sensenbaugh \& Sanders, 1999; Wang et al., 1989), it is not clear what regulatory sequences of the ovalbumin promoter are sufficient and efficient enough for inducing oviduct-specific expression of exogenous genes in the bioreactor chickens. In plasmid constructs, various lengths of chicken ovalbumin promoter fragments and, 5' and 3'

flanking regions have been fused to the exogenous genes in order to induce gene expression. Some reports suggest that the inclusion of two major regulatory elements residing in the chicken ovalbumin promoter, a steroid-dependent regulatory element (SDRE, -900 to -732) and a negative regulatory element (NRE, -308 to -88) is sufficient to induce oviduct-specific expression of a therapeutic protein (S. C. Kwon et al., 2010; S G Lillico et al., 2007). These two regulatory elements are critical for appropriate regulation of Ovalbumin gene expression (Gaub, Dierich, Astinotti, Touitou, \& Chambon, 1987; Nordstrom, Dean, \& Sanders, 1993; Sanders \& McKnight, 1988; Schweers et al., 1990; Schweers \& Sanders, 1991). The SDRE is required for responsiveness to steroid hormones (i.e., estrogen, progesterone, androgen, and glucocorticoids) (Schimke et al., 1975) and the NRE, acts as a bifunctional element, cooperating with SDRE to activate Ovalbumin gene expression in the presence of steroids in the oviduct tissue, and repressing the Ovalbumin gene transcription in the absence of steroids in the oviduct and non-oviduct cells (Gaub et al., 1987; Haecker et al., 1995; Sanders \& McKnight, 1988; Sensenbaugh \& Sanders, 1999).

In an attempt to improve the expression level of the transgene ex situ (out of the native genomic context), additional regulatory sequences comprising the ovalbumin exon 1, intron 1 , and the beginning of exon 2 were included in the promoter construct (S G Lillico et al., 2007). Zhu et al. utilized either $7.5 \mathrm{~kb}$ and 
$15 \mathrm{~kb}$ of the 5 ' flanking region, and $15.5 \mathrm{~kb}$ of the 3' flanking region from the Ovalbumingene to direct transgene expression ex situ. Although these regions contained all oviduct-specific regulatory elements, the ectopic expression of the transgene was detected in non-oviduct tissue of the chimeric chicken, and also germline transmission did not occur under the conditions of this study (Zhu et al., 2005). In the other studies, it was assumed that inclusion of the estrogen-responsive enhancer element (ERE), normally located approximately $3.3 \mathrm{~kb}$ upstream from the transcription start site (Figure 1A) (Kato et al., 1992) in the ovalbumin promoter-driven construct would increase the expression level of transgene (M. S. Kwon et al., 2018; S G Lillico et al., 2007). On the contrary, the results of the study failed to prove any increase in the level of recombinant protein produced in the transgenic chickens (S G Lillico et al., 2007). Herron et al. reintroduced an additional regulatory sequence between ERE and SDRE in their construct to enhance the expression level of protein in the egg white (Herron et al., 2018). The ovalbumin promoter (ranging from $1.35 \mathrm{~kb}$ to $3.0 \mathrm{~kb}$ ) which have been used in most of ex situ (in a non-native site of the genome, or in a plasmid construct) studies so far, contains five main conserved sites which have been identified in chicken and other avian species (Woodfint, Hamlin, \& Lee, 2018). However, the progressive identification of other farther regulatory elements associated with oviduct specificity (Kodama et al., 2012) and the complexity of gene expression regulation, have inevitably led to the use of ovalbumin promoter in situ (in its original genomic position).

Oishi et al. were the first and remain the only group to report the successful pruduction of pharmaceutical proteins driven by endogenous ovalbumin promoter in the egg white of transgenic chickens (Oishi et al., 2018). Low number of reports is due to the challenges in the generation of transgenic chickens. Although transgenic chicken bioreactors are valuable tools for the production of human recompinant proteins containing appropriate posttranslational modifications, generating of founder transgenic chicken is relatively difficult, inefficient and time consuming. Thus, the use of alternative cell production systems, for example chicken non-oviduct cell lines, would seem desirable to overcome these obstacles.

Previous studies on the precise characterization of the regulatory properties of the Ovalbumingene demonstrated that deletion of the SDRE and the NRE, as well as the linker between them, increases chloramphenicol acetyltransferase (CAT) activity on a plasmid (Haecker et al., 1995; Sanders \& McKnight, 1988; Sensenbaugh \& Sanders, 1999). These studies indicated that a cooperation between multiple distal regulatory and promoter-proximal regions confers oviduct-specific Ovalbuminexpression. Deletion of regulatory elements upstream of -80 abolished the tissue-specific expression of Ovalbumin in primary oviduct cell cultures, while basal expression increased to levels seen with estrogen-induced genes containing a SDRE (Haecker et al., 1995; H. M. Park et al., 2000; Sanders \& McKnight, 1988). A few reports showed that the expression of the reporter CAT gene was induced by the ovalbumin proximal promoter $(-87$ to +9$)$ in primary oviduct cell and non-oviduct cell cultures such as LMH/2A (Table 3) (Dean, Jones, \& Sanders, 1996; Haecker et al., 1995; Monroe \& Sanders, 2000; Muramatsu et al., 1998; H. M. Park et al., 2000; Schweers et al., 1990; Sensenbaugh \& Sanders, 1999).

Although previous transfection experiments with truncated ovalbumin promoter-CAT reporter (OvCAT) constructs have tried to mimic the activity of the endogenous ovalbumin promoter in the oviduct and nonoviduct cells, there is not any report on the in situ deletion of the regulatory sequences of ovalbumin promoter and thier effects on the levels of Ovalbumin gene expression. In this study, we show that thein situ deletion of distal ovalbumin promoter results in the upregulation of Ovalbumin transcript in chicken DF1 cell line. Our RT-qPCR analysis upon deletion of the distal ovalbumin promoter including two major regulatory elements, the SDRE and the NRE (DF1 ${ }^{+/ O V A}$ Pro [?] cells), indicated an increased level of expression of ovalbumin, $\sim 10^{4}$ fold higher than the Ovalbumin transcript levels in WT DF1 (Figures 2). Deletion of a 962bp region (-1044 to $-82 \mathrm{bp}$ ) containing the distal promoter elements completely abolished tissue-restricted and hormone-dependent expression of the Ovalbumin gene. It has been reported that chicken ovalbumin upstream promoter (COUP) site (-85 to -73) represses basal Ovalbumin expression in the absent of steroids and is required for induction by steroids (Figure 1A) (H. M. Park et al., 2000). Although previous reports have shown that the deletion of the COUP site in OvCAT constructs increases transcriptional activity in the absence of the NRE and confirm its repression role on the basal gene expression, our data clearly show 
that, without the NRE, transcriptional activity is increased even when COUP site is present. This finding suggests that the opposing effect of COUP site on the transcriptional activity depends on the native genomic context and perhaps to other regulatory elements in wild-type composition.

In our DF1+/OVA Pro [?] cells, althogh the core promoter elements (TATA box and the initiator element (INR)containing sufficient information for the initiation of transcription) have been remained intact, we cannot rule out the regulatory role of alternative promoters in the genome (Ayoubi \& Van De Ven, 1996). Kodama et al. have found several TATA-like and other promoter motifs located at a position around -1800 bp (Kodama et al., 2012). Muramatsu et al. demonstrated that the sequence from -3200 to -2800 act as a tissue-specific silencer-like which represses the Ovalbumin gene expression in non-oviduct tissue (Figure 1A) (Muramatsu et al., 1998). However, the presence of this sequence did not inhibit the transcriptional activity under our experimental conditions. Our results support this notion that the transcriptional regulation is not determined only by promoter regions, but involves multiple native features in the local genomic context including enhancers, insulators, DNA binding regulatory proteins such as transcription factors and repressors, nucleosome positioning, histone modifications, non-coding RNA, the three-dimensional organization of genes, and epigenetic mechanisms (Andersson \& Sandelin, 2019; Gibcus \& Dekker, 2012).

In conclusion, our study demonstrates the potential for producing recombinant proteins in chicken cell lines as an appropriate alternative to mammalian cell culture systems. This accomplishment of hormonallyindependent expression of the transgene driven by the endogenous regulatory mechanism(s) overcomes the limitation of cloned promoters, where the promoter regulatory sequences have to be taken out of their cis context and spatial organization into a plasmid. Use of CRISPR technology enables precise deletion or mutagenesis of regulatory sequences in the native genomic context, showing great promise to better understand, regulate, and exploit the native biological elements of gene regulation.

\section{ACKNOWLEDGMENTS}

We dedicate this work to our deceased colleague, Dr. Mohammad Reza Bassami, for his help to conceptualize the early stages of this study.

\section{AUTHOR CONTRIBUTIONS STATEMENT}

Conceived and designed the experiments: HD and SY. Performed the experiments: SY, ND, and LG. Analyzed the data: SY and HD. Acted as thesis advisor: JM. Supervised the project and experiments: HD. Wrote the paper: SY and HD. All authors have read and confirmed the contents of the manuscript.

\section{CONFLICT OF INTERESTS}

The authors declare that they have no conflict of interest.

\section{FUNDING}

This work was supported by grant number 942331 from Ferdowsi University of Mashhad. Funding for open access charge: Ferdowsi University of Mashhad. 


\section{REFERENCES}

Abu-Bonsrah, K. D., Zhang, D., \& Newgreen, D. F. (2016). CRISPR/Cas9 Targets Chicken Embryonic Somatic Cells In Vitro and In Vivo and generates Phenotypic Abnormalities. Scientific Reports , 6 , 34524. https://doi.org/10.1038/srep34524

Andersson, R., \& Sandelin, A. (2019). Determinants of enhancer and promoter activities of regulatory elements. Nature Reviews Genetics . https://doi.org/10.1038/s41576-019-0173-8

Ayoubi, T. A., \& Van De Ven, W. J. (1996). Regulation of gene expression by alternative promoters. FASEB Journal : Official Publication of the Federation of American Societies for Experimental Biology , 10 (4), 453-460.

Bellard, M., Dretzen, G., Bellard, F., Oudet, P., \& Chambon, P. (1982). Disruption of the typical chromatin structure in a 2500 base-pair region at the 5' end of the actively transcribed ovalbumin gene. The EMBO Journal , 1 (2), 223-230.

Byun, S. J., Kim, S. W., Kim, K.-W., Kim, J. S., Hwang, I.-S., Chung, H. K., . . Y Yoo, J. G. (2011). Oviductspecific enhanced green fluorescent protein expression in transgenic chickens. Bioscience, Biotechnology, and Biochemistry , 75 (4), 646-649. https://doi.org/10.1271/bbb.100721

Cao, D., Wu, H., Li, Q., Sun, Y., Liu, T., Fei, J., ... Li, N. (2015). Expression of recombinant human lysozyme in egg whites of transgenic hens. PloS One , 10 (2), e0118626. https://doi.org/10.1371/journal.pone.0118626

Dean, D. M., Jones, P. S., \& Sanders, M. M. (1996). Regulation of the chicken ovalbumin gene by estrogen and corticosterone requires a novel DNA element that binds a labile protein, Chirp-1. Molecular and Cellular Biology , 16 (5), 2015-2024. https://doi.org/10.1128/mcb.16.5.2015

Demain, A. L., \& Vaishnav, P. (2009). Production of recombinant proteins by microbes and higher organisms. Biotechnology Advances , 27 (3), 297-306. https://doi.org/10.1016/j.biotechadv.2009.01.008

Dougherty, D. C., Park, H.-M., \& Sanders, M. M. (2009). Interferon regulatory factors (IRFs) repress transcription of the chicken ovalbumin gene. Gene , 439 (1-2), 63-70. https://doi.org/10.1016/j.gene.2009.03.016

Dougherty, D. C., \& Sanders, M. M. (2005). Estrogen action: revitalization of the chick oviduct model. Trends in Endocrinology and Metabolism: TEM , 16 (9), 414-419. https://doi.org/10.1016/j.tem.2005.09.001

Elliott, S., Egrie, J., Browne, J., Lorenzini, T., Busse, L., Rogers, N., \& Ponting, I. (2004). Control of rHuEPO biological activity: the role of carbohydrate. Experimental Hematology , 32 (12), 1146-1155. https://doi.org/10.1016/j.exphem.2004.08.004

Gaub, M. P., Dierich, A., Astinotti, D., Touitou, I., \& Chambon, P. (1987). The chicken ovalbumin promoter is under negative control which is relieved by steroid hormones. The EMBO Journal , 6 (8), 2313-2320.

Gibcus, J. H., \& Dekker, J. (2012). The context of gene expression regulation. F1000 Biology Reports , 4 (1). https://doi.org/10.3410/B4-8

Haecker, S. A., Muramatsu, T., Sensenbaugh, K. R., \& Sanders, M. M. (1995). Repression of the ovalbumin gene involves multiple negative elements including a ubiquitous transcriptional silencer.Molecular Endocrinology (Baltimore, Md.) , 9 (9), 1113-1126. https://doi.org/10.1210/mend.9.9.7491104

Herron, L. R., Pridans, C., Turnbull, M. L., Smith, N., Lillico, S., Sherman, A., .. Sang, H. M. (2018). A chicken bioreactor for efficient production of functional cytokines. BMC Biotechnology ,18 (1), 82. https://doi.org/10.1186/s12896-018-0495-1 
Houdebine, L.-M. (2018). Production of pharmaceutical proteins by transgenic animals. Revue Scientifique et Technique (International Office of Epizootics) , 37 (1), 131-139. https://doi.org/10.20506/rst.37.1.2746

Ivarie, R. (2003). Avian transgenesis: progress towards the promise. Trends in Biotechnology , 21 (1), 14-19.

Ivarie, R. (2006). Competitive bioreactor hens on the horizon. Trends in Biotechnology , 24 (3), 99-101. https://doi.org/10.1016/j.tibtech.2006.01.004

Kato, S., Tora, L., Yamauchi, J., Masushige, S., Bellard, M., \& Chambon, P. (1992). A far upstream estrogen response element of the ovalbumin gene contains several half-palindromic 5'-TGACC-3' motifs acting synergistically. Cell , 68 (4), 731-742. https://doi.org/10.1016/0092-8674(92)90148-6

Kaye, J. S., Bellard, M., Dretzen, G., Bellard, F., \& Chambon, P. (1984). A close association between sites of DNase I hypersensitivity and sites of enhanced cleavage by micrococcal nuclease in the 5'-flanking region of the actively transcribed ovalbumin gene. The EMBO Journal , 3 (5), 1137-1144.

Kaye, J. S., Pratt-Kaye, S., Bellard, M., Dretzen, G., Bellard, F., \& Chambon, P. (1986). Steroid hormone dependence of four DNase I-hypersensitive regions located within the 7000-bp 5'-flanking segment of the ovalbumin gene. The EMBO Journal , 5 (2), 277-285.

Kodama, D., Nishimiya, D., Iwata, K.-I., Yamaguchi, K., Yoshida, K., Kawabe, Y., .. Iijima, S. (2008). Production of human erythropoietin by chimeric chickens. Biochemical and Biophysical Research Communications , 367 (4), 834-839. https://doi.org/10.1016/j.bbrc.2008.01.020

Kodama, D., Nishimiya, D., Nishijima, K.-I., Okino, Y., Inayoshi, Y., Kojima, Y., ... Iijima, S. (2012). Chicken oviduct-specific expression of transgene by a hybrid ovalbumin enhancer and the Tet expression system. Journal of Bioscience and Bioengineering ,113 (2), 146-153. https://doi.org/10.1016/j.jbiosc.2011.10.006

Kwon, M. S., Koo, B. C., Kim, D., Nam, Y. H., Cui, X.-S., Kim, N.-H., \& Kim, T. (2018). Generation of transgenic chickens expressing the human erythropoietin (hEPO) gene in an oviduct-specific manner: Production of transgenic chicken eggs containing human erythropoietin in egg whites.PloS One, 13 (5), e0194721. https://doi.org/10.1371/journal.pone.0194721

Kwon, S. C., Choi, J. W., Jang, H.-J., Shin, S. S., Lee, S. K., Park, T. S., .. Han, J. Y. (2010). Production of biofunctional recombinant human interleukin 1 receptor antagonist (rhIL1RN) from transgenic quail egg white. Biology of Reproduction , 82 (6), 1057-1064. https://doi.org/10.1095/biolreprod.109.081687

Lillico, S G, Sherman, A., McGrew, M. J., Robertson, C. D., Smith, J., Haslam, C., ... Sang, H. M. (2007). Oviduct-specific expression of two therapeutic proteins in transgenic hens. Proceedings of the National Academy of Sciences of the United States of America ,104 (6), 1771-1776. https://doi.org/10.1073/pnas.0610401104

Lillico, Simon G., McGrew, M. J., Sherman, A., \& Sang, H. M. (2005). Transgenic chickens as bioreactors for protein-based drugs. Drug Discovery Today, Vol. 10, pp. 191-196. https://doi.org/10.1016/S13596446(04)03317-3

Liu, T., Wu, H., Cao, D., Li, Q., Zhang, Y., Li, N., \& Hu, X. (2015). Oviduct-specific expression of human neutrophil defensin 4 in lentivirally generated transgenic chickens. PloS One,10 (5), e0127922. https://doi.org/10.1371/journal.pone.0127922

Liu, Z., Tyo, K. E. J., Martinez, J. L., Petranovic, D., \& Nielsen, J. (2012). Different expression systems for production of recombinant proteins in Saccharomyces cerevisiae. Biotechnology and Bioengineering, 109 (5), 1259-1268. https://doi.org/10.1002/bit.24409

Maksimenko, O. G., Deykin, A. V, Khodarovich, Y. M., \& Georgiev, P. G. (2013). Use of transgenic animals in biotechnology: prospects and problems. Acta Naturae , 5 (1), 33-46. 
Monroe, D. G., \& Sanders, M. M. (2000). The COUP-adjacent repressor (CAR) element participates in the tissue-specific expression of the ovalbumin gene. Biochimica et Biophysica Acta , 1517 (1), 27-32. https://doi.org/10.1016/s0167-4781(00)00241-4

Muramatsu, T., Imai, T., Park, H. M., Watanabe, H., Nakamura, A., \& Okumura, J. I. (1998). Gene gunmediated in vivo analysis of tissue-specific repression of gene transcription driven by the chicken ovalbumin promoter in the liver and oviduct of laying hens.Molecular and Cellular Biochemistry , 185 (1-2), 27-32. https://doi.org/10.1023/A:1016507900718

Nordstrom, L. A., Dean, D. M., \& Sanders, M. M. (1993). A complex array of double-stranded and singlestranded DNA-binding proteins mediates induction of the ovalbumin gene by steroid hormones. The Journal of Biological Chemistry, 268 (18), 13193-13202.

Oishi, I., Yoshii, K., Miyahara, D., \& Tagami, T. (2018). Efficient production of human interferon beta in the white of eggs from ovalbumin gene-targeted hens. Scientific Reports , 8 (1), 10203. https://doi.org/10.1038/s41598-018-28438-2

Park, H. M., Haecker, S. E., Hagen, S. G., \& Sanders, M. M. (2000). COUP-TF plays a dual role in the regulation of the ovalbumin gene.Biochemistry , 39 (29), 8537-8545. https://doi.org/10.1021/bi0005862

Park, T. S., Lee, H. G., Moon, J. K., Lee, H. J., Yoon, J. W., Yun, B. N. R., .. . Han, B. K. (2015). Deposition of bioactive human epidermal growth factor in the egg white of transgenic hens using an oviduct-specific minisynthetic promoter. FASEB Journal : Official Publication of the Federation of American Societies for Experimental Biology , 29 (6), 2386-2396. https://doi.org/10.1096/fj.14-264739

Pfaffl, M. W. (2001). A new mathematical model for relative quantification in real-time RT-PCR. Nucleic Acids Research ,29 (9), e45. https://doi.org/10.1093/nar/29.9.e45

Raju, T. S., Briggs, J. B., Borge, S. M., \& Jones, A. J. (2000). Species-specific variation in glycosylation of IgG: evidence for the species-specific sialylation and branch-specific galactosylation and importance for engineering recombinant glycoprotein therapeutics.Glycobiology , 10 (5), 477-486. https://doi.org/10.1093/glycob/10.5.477

Rapp, J. C., Harvey, A. J., Speksnijder, G. L., Hu, W., \& Ivarie, R. (2003). Biologically active human interferon alpha-2b produced in the egg white of transgenic hens. Transgenic Research , 12 (5), 569-575.

Sanders, M. M., \& McKnight, G. S. (1988). Positive and negative regulatory elements control the steroidresponsive ovalbumin promoter.Biochemistry , 27 (17), 6550-6557. https://doi.org/10.1021/bi00417a053

Schimke, R. T., McKnight, G. S., Shapiro, D. J., Sullivan, D., \& Palacios, R. (1975). Hormonal regulation of ovalbumin synthesis in the chick oviduct. Recent Progress in Hormone Research , 31 , 175-211.

Schweers, L. A., Frank, D. E., Weigel, N. L., \& Sanders, M. M. (1990). The steroid-dependent regulatory element in the ovalbumin gene does not function as a typical steroid response element. The Journal of Biological Chemistry, 265 (13), 7590-7595.

Schweers, L. A., \& Sanders, M. M. (1991). A protein with a binding specificity similar to NF-kappa B binds to a steroid-dependent regulatory element in the ovalbumin gene. The Journal of Biological Chemistry , 266 (16), 10490-10497.

Sensenbaugh, K. R., \& Sanders, M. M. (1999). Multiple promoter elements including a novel repressor site modulate expression of the chick ovalbumin gene. DNA and Cell Biology , 18 (2), 147-156. https://doi.org/10.1089/104454999315538

T. Shantha Raju. (2003). No Title. Bioprocess International, 1, . , 1 , 44-53.

Wang, L. H., Tsai, S. Y., Cook, R. G., Beattie, W. G., Tsai, M. J., \& O’Malley, B. W. (1989). COUP transcription factor is a member of the steroid receptor superfamily. Nature , 340 (6229), 163-166. https://doi.org/10.1038/340163a0 
Woodfint, R. M., Hamlin, E., \& Lee, K. (2018). Avian Bioreactor Systems: A Review. Molecular Biotechnology , 60 (12), 975-983. https://doi.org/10.1007/s12033-018-0128-x

Yoshimura, M., \& Oka, T. (1990). Transfection of beta-casein chimeric gene and hormonal induction of its expression in primary murine mammary epithelial cells. Proceedings of the National Academy of Sciences of the United States of America , 87 (10), 3670-3674. https://doi.org/10.1073/pnas.87.10.3670

Zhu, L., van de Lavoir, M.-C., Albanese, J., Beenhouwer, D. O., Cardarelli, P. M., Cuison, S., ... Etches, R. J. (2005). Production of human monoclonal antibody in eggs of chimeric chickens. Nature Biotechnology , 23 (9), 1159-1169. https://doi.org/10.1038/nbt1132

\section{FIGURE LEGENDS}

Figure 1. Design and validation of the targeted deletion of ovalbumin distal promoter elements in DF1 cells.

1. The schematic representation of CRISPR/Cas9 mediated deletion strategy of the ovalbumin promoter in DF1 cells. The top diagram shows the wild-type (WT) chicken ovalbumin locus. The two guide RNA (gRNA 1 and 2) binding sites are shown by red bars below the regulatory sequences of the ovalbumin promoter. The gRNAs 1 and 2 target two positions downstream of NRE (downstream of CAR; COUPadjacent repressor site in the negative regulatory element) and upstream of SDRE, respectively. The bottom diagram shows the locus after CRISPR-mediated deletion of the distal ovalbumin promoter in DF1 cells. The PCR primers used for the assessment of deletion (P1 to P3), and the ovalbumin gene expression (P4 and P5, used in Figure 2) are shown as small arrows.

2. Two-step genomic PCR to confirm the deletion of distal promoter of the ovalbumin gene. In the first PCR (using P1 and P3 primers), an amplicon of $1310 \mathrm{bp}$ was acquired from the wild-type (WT) allele. In a hemi-nested PCR (using P1 and P2 primers), amplicons of $1256 \mathrm{bp}$ and $~ 316 \mathrm{bp}$ were acquired from the wild-type and promoter-deleted (DF1 [?]) alleles, respectively.

3. Alignment of the representative sequences of the wild-type (WT DF1) and promoter-deleted (DF1 $\left.{ }^{[?]}\right)$ sequences acquired by Sanger sequencing. The gRNA-binding sites regions are shown in blue, and the PAM regions are shown in green letters. WT, wild type; $\mathrm{DF} 1^{[?]}$, distal ovalbumin promoter knockout DF1 cells (DF1+/OVA Pro [?]); NHEJ, non-homologous end-joining; ERE, estrogen-responsive enhancer element; TSSL, tissue-specific silencer-like element; SDRE, steroid-dependent regulatory element; NRE, negative regulatory element; CAR, COUP-adjacent repressor site; COUP, Chicken ovalbumin upstream promoter; TATA, TATA box; INR, Initiator element; P, primer. M, DNA size marker; NTC, no template control.

Figure 2. Gene expression ratio for ovalbumin transcript in DF1+/OVA Pro [?] cells.

1. Up- regulation of the ovalbumin mRNA in DF1 +/OVA Pro $\left[{ }^{[?]}\right.$ cells were assessed by RT-qPCR. Upon deletion of the distal ovalbumin promoter, an increased level of expression of ovalbumin was determined $\left(\mathrm{DF} 1{ }^{[?]}\right)$. The transcript levels of ovalbumin for these samples are $\sim 10^{4}$ fold higher than the ovalbumin transcript levels in the wild-type DF1 (WT DF1). The transcript levels for ovalbumin in the hormonally-activated tissue of magnum (from 35-week old laying hen) shows the highest level of expression. Gene expression ratio for the Ovalbumin gene over Gapdh has been calculated by the Pfaffl method of relative quantification.

2. Agarose gel (2\%) electrophoresis for analysis of the RT-PCR products amplified by primers P4 and P5 (for ovalbumin, Figure 1 and Table 2), and P8 and P9 (for Gapdh, Table 2). The expected amplicon size for ovalbumin and Gapdh are $179 \mathrm{bp}$ and $187 \mathrm{bp}$, respectively. WT, wild type; DF1 [?], distal ovalbumin promoter knockout DF1 cells (DF1 +/OVA Pro [?]); M, DNA size marker; NTC, no template control. RT, reverse transcriptase.

Figure 3. Activation of transgene expression in DF1+/OVA Pro [?]-Tg (promoterless dsRed) cells.

1. The schematic representation of CRISPR HDR mediated knockin strategy in DF1+/OvA Pro [?] cells. The top diagram shows the donor vector that designed to have a promoterless DsRed2 and a CMV- 
Puro-EGFP cassette flanked by left and right homology arms. The gRNA3 (denoted by the red bar) indicates the gRNA-binding site on exon 2 of the ovalbumin $(+174$ to +1784$)$ gene. The bottom diagram shows the allele after CRISPR-HDR insertion of the reporter cassette (DF1 $1^{\text {[?]-Tg }}$ cells). PCR primers (P6 and P7) were used for the assessment of the CRISPR-HDR insertion of the promoterless DsRed2 in DF1 [?]-Tg cells.

2. Genomic PCR analysis of the targeted gene knockin DF1 ${ }^{[?]-T g}$ cells. For the assessment of the CRISPRHDR insertion of the promoterless DsRed2 in DF1 ${ }^{[?]-{ }^{-T g}}$ cells, primers (P6 and P7) were used to amplify a 2569 bp amplicon in DF1 [?]-Tg cells.

3. The insertion-specific PCR product of DF1 [?]-Tg cells was sequenced by Sanger sequencing and aligned to the donor plasmid (used as a DNA repair template during transfection).

4. Fluorescence microscopy of DF1 [?]-Tg cells indicating DsRed2 expression under the control of the endogenous truncated ovalbumin promoter. DF1 [?], distal ovalbumin promoter knock-out DF1 cells (DF1 +/OVA Pro [?] $)$ DF1 $1^{[?]-T g}$ cells, promoterless DsRed2 knockin DF1 cells (DF1 +/OVA Pro [?]-Tg (promoterless dsRed) ); HDR, homology-directed repair; M, DNA size marker; WT, wild type; NTC, no template control.

Figure 4. Graphical abstract depicting the increased expression of the Ovalbumin gene and the induced activity of an inserted transgene after CRISPR/CAS-mediated deletion of the regulatory sequences of the ovalbumin promoter.

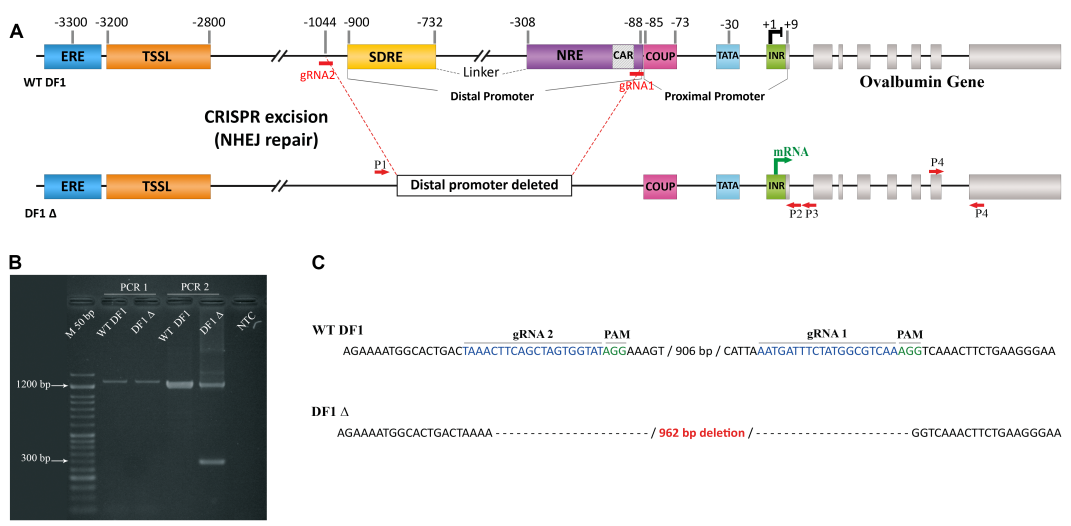

A

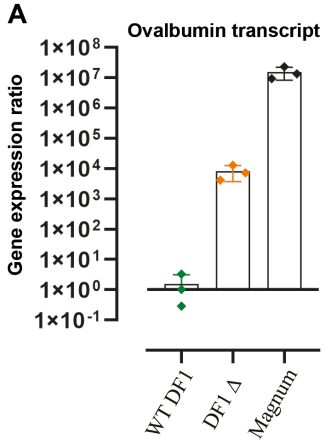

B

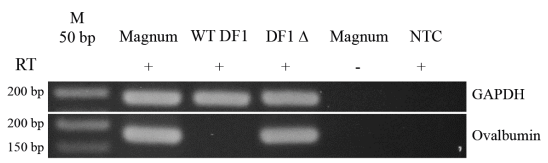




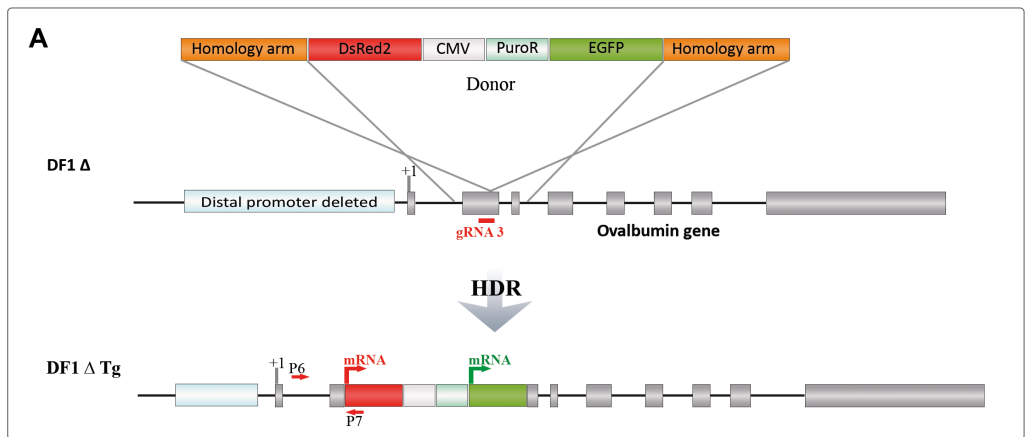

B

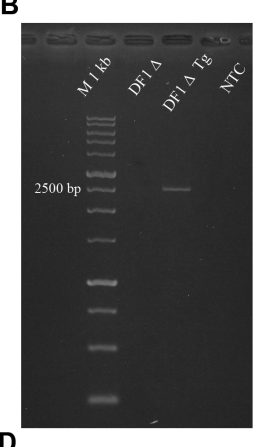

C
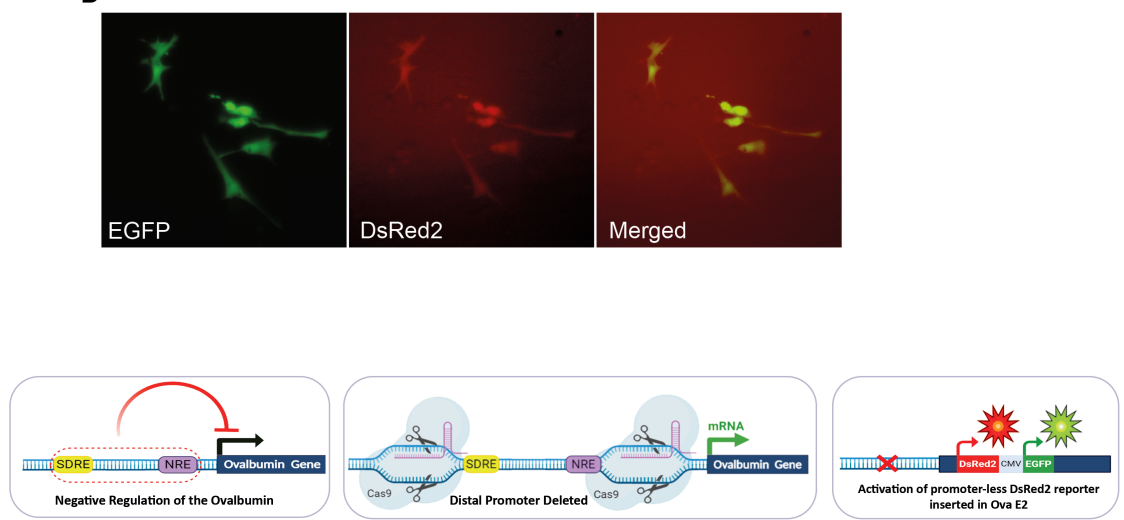

Negative Regulation of the Ovalbumin

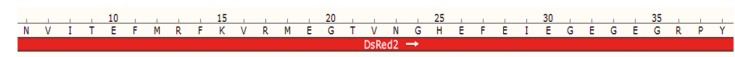

AACGTCATCACCGAGTTCATOCGCTTCAAGGTOCOCATGGAGGOCACCGTOAACGOCCACGAGTTCGAGATCOAGGOCGAGGQCGAGGOCCOCCCCTAC

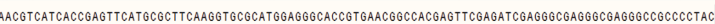

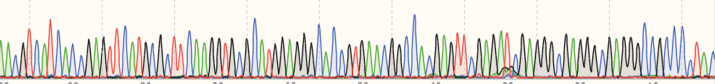

DsRed2

Merged

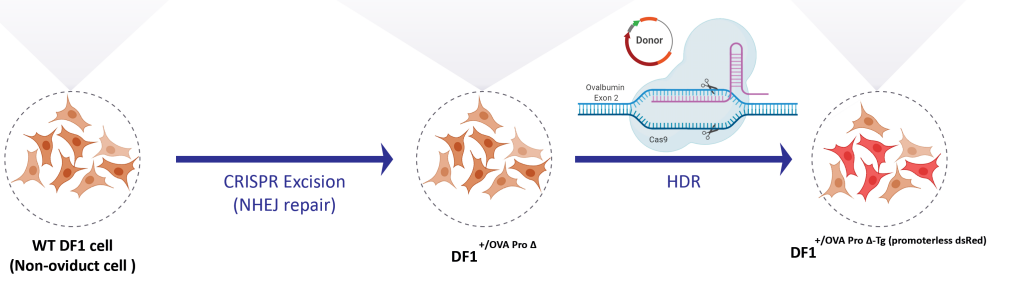

Ovalbumin Transcript Levels

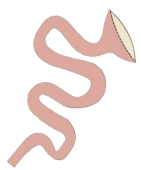

Immature magnum tissue (In the absence of estrogen )

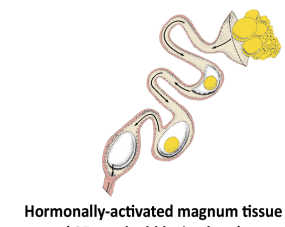

(35-week old laying hen ) 